MATHEMATICS OF COMPUTATION

Volume 67, Number 222, April 1998, Pages 647-665

S 0025-5718(98)00925-9

\title{
APPROXIMATION PROPERTIES OF MULTIVARIATE WAVELETS
}

\author{
RONG-QING JIA
}

\begin{abstract}
Wavelets are generated from refinable functions by using multiresolution analysis. In this paper we investigate the approximation properties of multivariate refinable functions. We give a characterization for the approximation order provided by a refinable function in terms of the order of the sum rules satisfied by the refinement mask. We connect the approximation properties of a refinable function with the spectral properties of the corresponding subdivision and transition operators. Finally, we demonstrate that a refinable function in $W_{1}^{k-1}\left(\mathbb{R}^{s}\right)$ provides approximation order $k$.
\end{abstract}

\section{INTRODUCTION}

We are concerned with functional equations of the form

$$
\phi=\sum_{\alpha \in \mathbb{Z}^{s}} a(\alpha) \phi(M \cdot-\alpha),
$$

where $\phi$ is the unknown function defined on the $s$-dimensional Euclidean space $\mathbb{R}^{s}$, $a$ is a finitely supported sequence on $\mathbb{Z}^{s}$, and $M$ is an $s \times s$ integer matrix such that $\lim _{n \rightarrow \infty} M^{-n}=0$. The equation (1.1) is called a refinement equation, and the matrix $M$ is called a dilation matrix. Correspondingly, the sequence $a$ is called the refinement mask. Any function satisfying a refinement equation is called a refinable function.

If $a$ satisfies

$$
\sum_{\alpha \in \mathbb{Z}^{s}} a(\alpha)=m:=|\operatorname{det} M|,
$$

then it is known that there exists a unique compactly supported distribution $\phi$ satisfying the refinement equation (1.1) subject to the condition $\hat{\phi}(0)=1$. This distribution is said to be the normalized solution to the refinement equation with mask $a$. This fact was essentially proved by Cavaretta, Dahmen, and Micchelli in [7, Chap. 5] for the case in which the dilation matrix is 2 times the $s \times s$ identity matrix $I$. The same proof applies to the general refinement equation (1.1).

Wavelets are generated from refinable functions. In [20], Jia and Micchelli discussed how to construct multivariate wavelets from refinable functions associated

Received by the editor April 17, 1996.

1991 Mathematics Subject Classification. Primary 41A25, 41A63; Secondary 42C15, 65D15.

Key words and phrases. Refinement equations, refinable functions, wavelets, accuracy, approximation order, smoothness, subdivision operators, transition operators.

Supported in part by NSERC Canada under Grant OGP 121336.

(C) 1998 American Mathematical Society 
with a general dilation matrix. The approximation and smoothness properties of wavelets are determined by the corresponding refinable functions.

In [9], DeVore, Jawerth, and Popov established a basic theory for nonlinear approximation by wavelets. In their work, the refinement mask was required to be nonnegative. In [15], Jia extended their results and, in particular, removed the restriction of non-negativity of the mask.

Our goal is to characterize the approximation order provided by a refinable function in terms of the refinement mask. This information is important for our understanding of wavelet approximation.

Before proceeding further, we introduce some notation. A multi-index is an $s$ tuple $\mu=\left(\mu_{1}, \ldots, \mu_{s}\right)$ with its components being nonnegative integers. The length of $\mu$ is $|\mu|:=\mu_{1}+\cdots+\mu_{s}$, and the factorial of $\mu$ is $\mu !:=\mu_{1} ! \cdots \mu_{s} !$. For two multi-indices $\mu=\left(\mu_{1}, \ldots, \mu_{s}\right)$ and $\nu=\left(\nu_{1}, \ldots, \nu_{s}\right)$, we write $\nu \leq \mu$ if $\nu_{j} \leq \mu_{j}$ for $j=1, \ldots, s$. If $\nu \leq \mu$, then we define

$$
\left(\begin{array}{l}
\mu \\
\nu
\end{array}\right):=\frac{\mu !}{\nu !(\mu-\nu) !}
$$

For $j=1, \ldots, s, D_{j}$ denotes the partial derivative with respect to the $j$ th coordinate. For $\mu=\left(\mu_{1}, \ldots, \mu_{s}\right), D^{\mu}$ is the differential operator $D_{1}^{\mu_{1}} \cdots D_{s}^{\mu_{s}}$. Moreover, $p_{\mu}$ denotes the monomial given by

$$
p_{\mu}(x):=x_{1}^{\mu_{1}} \cdots x_{s}^{\mu_{s}}, \quad x=\left(x_{1}, \ldots, x_{s}\right) \in \mathbb{R}^{s} .
$$

The total degree of $p_{\mu}$ is $|\mu|$. For a nonnegative integer $k$, we denote by $\Pi_{k}$ the linear span of $\left\{p_{\mu}:|\mu| \leq k\right\}$. Then $\Pi:=\bigcup_{k=0}^{\infty} \Pi_{k}$ is the linear space of all polynomials of $s$ variables. We agree that $\Pi_{-1}=\{0\}$.

The Fourier transform of an integrable function $f$ on $\mathbb{R}^{s}$ is defined by

$$
\hat{f}(\xi)=\int_{\mathbb{R}^{s}} f(x) e^{-i x \cdot \xi} d x, \quad \xi \in \mathbb{R}^{s},
$$

where $x \cdot \xi$ denotes the inner product of two vectors $x$ and $\xi$ in $\mathbb{R}^{s}$. The domain of the Fourier transform can be naturally extended to include compactly supported distributions.

We denote by $\ell\left(\mathbb{Z}^{s}\right)$ the linear space of all sequences on $\mathbb{Z}^{s}$, and by $\ell_{0}\left(\mathbb{Z}^{s}\right)$ the linear space of all finitely supported sequences on $\mathbb{Z}^{s}$. For $\alpha \in \mathbb{Z}^{s}$, we denote by $\delta_{\alpha}$ the element in $\ell_{0}\left(\mathbb{Z}^{s}\right)$ given by $\delta_{\alpha}(\alpha)=1$ and $\delta_{\alpha}(\beta)=0$ for all $\beta \in \mathbb{Z}^{s} \backslash\{\alpha\}$. In particular, we write $\delta$ for $\delta_{0}$. For $j=1, \ldots, s$, let $e_{j}$ be the $j$ th coordinate unit vector. The difference operator $\nabla_{j}$ on $\ell\left(\mathbb{Z}^{s}\right)$ is defined by $\nabla_{j} a:=a-a\left(\cdot-e_{j}\right)$, $a \in \ell\left(\mathbb{Z}^{s}\right)$. For a multi-index $\mu=\left(\mu_{1}, \ldots, \mu_{s}\right), \nabla^{\mu}$ is the difference operator $\nabla_{1}^{\mu_{1}} \cdots \nabla_{s}^{\mu_{s}}$.

For a compactly supported distribution $\phi$ on $\mathbb{R}^{s}$ and a sequence $b \in \ell\left(\mathbb{Z}^{s}\right)$, the semi-convolution of $\phi$ with $b$ is defined by

$$
\phi *^{\prime} b:=\sum_{\alpha \in \mathbb{Z}^{s}} \phi(\cdot-\alpha) b(\alpha) .
$$

Let $\mathbb{S}(\phi)$ denote the linear space $\left\{\phi *^{\prime} b: b \in \ell\left(\mathbb{Z}^{s}\right)\right\}$. We call $\mathbb{S}(\phi)$ the shiftinvariant space generated by $\phi$. More generally, if $\Phi$ is a finite collection of compactly supported distributions on $\mathbb{R}^{s}$, then we use $\mathbb{S}(\Phi)$ to denote the linear space of all distributions of the form $\sum_{\phi \in \Phi} \phi *^{\prime} b_{\phi}$, where $b_{\phi} \in \ell\left(\mathbb{Z}^{s}\right)$ for $\phi \in \Phi$.

Here is a brief outline of the paper. In Section 2 we clarify the relationship between the order of approximation provided by $\mathbb{S}(\phi)$ and the accuracy of $\phi$, the 
order of the polynomial space contained in $\mathbb{S}(\phi)$. In Section 3 we introduce the socalled sum rules and give a characterization for the accuracy of a refinable function in terms of the order of the sum rules satisfied by the refinement mask. In Section 4, several examples are provided to illustrate the general theory. Section 5 is devoted to a study of the subdivision and transition operators and their applications to approximation properties of refinable functions. Finally, in Section 6, we show that a refinable function in $W_{1}^{k}\left(\mathbb{R}^{s}\right)$ associated with an isotropic dilation matrix has accuracy at least $k+1$.

\section{Approximation ORDER AND POLYNOMIAL REPRODUCIBILITY}

Let $\phi$ be a compactly supported function in $L_{p}\left(\mathbb{R}^{s}\right)(1 \leq p \leq \infty)$. In this section we clarify the relationship between the order of approximation provided by $\mathbb{S}(\phi)$ and the degree of the polynomial space contained in $\mathbb{S}(\phi)$. The reader is referred to [17] for a recent survey on approximation by shift-invariant spaces.

The norm in $L_{p}\left(\mathbb{R}^{s}\right)$ is denoted by $\|\cdot\|_{p}$. For an element $f \in L_{p}\left(\mathbb{R}^{s}\right)$ and a subset $G$ of $L_{p}\left(\mathbb{R}^{s}\right)$, the distance from $f$ to $G$, denoted by $\operatorname{dist}_{p}(f, G)$, is defined by

$$
\operatorname{dist}_{p}(f, G):=\inf _{g \in G}\|f-g\|_{p} .
$$

Let $S:=\mathbb{S}(\phi) \cap L_{p}\left(\mathbb{R}^{s}\right)$. For $h>0$, let $S^{h}:=\{g(\cdot / h): g \in S\}$. For a real number $\kappa \geq 0$, we say that $\mathbb{S}(\phi)$ provides approximation order $\kappa$ if for each sufficiently smooth function $f$ in $L_{p}\left(\mathbb{R}^{s}\right)$, there exists a constant $C>0$ such that

$$
\operatorname{dist}_{p}\left(f, S^{h}\right) \leq C h^{\kappa} \quad \forall h>0 .
$$

We say that $\mathbb{S}(\phi)$ provides density order $\kappa$ (see [3]) if for each sufficiently smooth function $f$ in $L_{p}\left(\mathbb{R}^{s}\right)$,

$$
\lim _{h \rightarrow 0} \operatorname{dist}_{p}\left(f, S^{h}\right) / h^{\kappa}=0 .
$$

Let $k$ be a positive integer. Suppose $\mathbb{S}(\phi) \supset \Pi_{k-1}$. Does $\mathbb{S}(\phi)$ always provide approximation order $k$ ? The answer is a surprising no. The first counterexample was given by de Boor and Höllig in [4] by considering bivariate $C^{1}$-cubics. Their results can be described in terms of box splines.

For a comprehensive study of box splines, the reader is referred to the book [5] by de Boor, Höllig, and Riemenschneider. For our purpose, it suffices to consider the box splines $M_{r, s, t}$ given by

$$
\widehat{M}_{r, s, t}(\xi)=\left(\frac{1-e^{-i \xi_{1}}}{i \xi_{1}}\right)^{r}\left(\frac{1-e^{-i \xi_{2}}}{i \xi_{2}}\right)^{s}\left(\frac{1-e^{-i\left(\xi_{1}+\xi_{2}\right)}}{i\left(\xi_{1}+\xi_{2}\right)}\right)^{t}, \quad \xi=\left(\xi_{1}, \xi_{2}\right) \in \mathbb{R}^{2},
$$

where $r, s$, and $t$ are nonnegative integers. It is easily seen that $M_{r, s, t} \in L_{\infty}\left(\mathbb{R}^{2}\right)$ if and only if $\min \{r+s, s+t, t+r\} \geq 1$. Let $\phi_{1}:=M_{2,1,2}$ and $\phi_{2}:=M_{1,2,2}$. In [4], de Boor and Höllig proved that $\mathbb{S}\left(\phi_{1}, \phi_{2}\right) \supseteq \Pi_{3}$ but $\mathbb{S}\left(\phi_{1}, \phi_{2}\right)$ does not provide $L_{\infty^{-}}$ approximation order 4 . In fact, the optimal $L_{\infty}$-approximation order provided by $\mathbb{S}\left(\phi_{1}, \phi_{2}\right)$ is 3 . In [21], Ron showed that there exists a compactly supported function $\psi$ in $\mathbb{S}\left(\phi_{1}, \phi_{2}\right)$ such that $\Pi_{3} \subseteq \mathbb{S}(\psi)$. Since $\mathbb{S}(\psi) \subseteq \mathbb{S}\left(\phi_{1}, \phi_{2}\right)$, the approximation order provided by $\mathbb{S}(\psi)$ is at most 3 .

In [6], de Boor and Jia extended the results in [4] in the following way. For $\rho=1,2, \ldots$, let $k$ be an integer such that $2 \rho+2 \leq k \leq 3 \rho+1$. Let

$$
\Phi:=\left\{M_{r, s, t} \in C^{\rho}\left(\mathbb{R}^{2}\right): r+s+t \leq k+2\right\} .
$$


Then $\mathbb{S}(\Phi) \supset \Pi_{k}$, but the optimal $L_{p}$-approximation order $(1 \leq p \leq \infty)$ provided by $\mathbb{S}(\Phi)$ is $k$, not $k+1$.

However, if $\mathbb{S}(\phi)$ provides approximation order $k$, then $\mathbb{S}(\phi)$ contains $\Pi_{k-1}$. This was proved by Jia in [16]. Under the additional condition that $\hat{\phi}(0) \neq 0$, it was proved by Ron $[21]$ that $\mathbb{S}(\phi)$ provides $L_{\infty}$-approximation order $k$ if and only if $\mathbb{S}(\phi)$ contains $\Pi_{k-1}$. In general, we have the following results, which were established in [16].

Theorem 2.1. Let $1 \leq p \leq \infty$, and let $\phi$ be a compactly supported function in $L_{p}\left(\mathbb{R}^{s}\right)$ with $\hat{\phi}(0) \neq 0$. For every positive integer $k$, the following statements are equivalent:

(a) $\mathbb{S}(\phi)$ provides approximation order $k$.

(b) $\mathbb{S}(\phi)$ provides density order $k-1$.

(c) $\mathbb{S}(\phi)$ contains $\Pi_{k-1}$.

(d) $D^{\mu} \hat{\phi}(2 \pi \beta)=0$ for all $\mu$ with $|\mu| \leq k-1$ and all $\beta \in \mathbb{Z}^{s} \backslash\{0\}$.

We remark that the implications $(\mathrm{a}) \Rightarrow(\mathrm{b}) \Rightarrow(\mathrm{c}) \Rightarrow(\mathrm{d})$ are valid without the assumption $\hat{\phi}(0) \neq 0$. Indeed, (a) $\Rightarrow$ (b) is obvious, (b) $\Rightarrow$ (c) was proved in [16], and the implication $(\mathrm{c}) \Rightarrow(\mathrm{d})$ was established in [2].

Suppose $\phi$ is the normalized solution of the refinement equation (1.1). If $\phi$ lies in $L_{p}\left(\mathbb{R}^{s}\right)$ for some $p, 1 \leq p \leq \infty$, then Theorem 2.1 applies to $\phi$, because $\hat{\phi}(0)=1$. Thus, there are two questions of interest. The first question is how to determine whether $\phi$ lies in $L_{p}\left(\mathbb{R}^{s}\right)$, and the second problem is how to characterize the highest degree of polynomials contained in $\mathbb{S}(\phi)$. The first question was discussed by Han and Jia in [12]. In this paper, we concentrate on the second question. When we speak of polynomial containment, $\phi$ is not required to be an integrable function. Thus, we say that a compactly supported distribution $\phi$ on $\mathbb{R}^{s}$ has accuracy $k$, if $\mathbb{S}(\phi) \supset \Pi_{k-1}$ (see [13] for the terminology of accuracy).

We point out that the equivalence between (c) and (d) in Theorem 2.1 remains true for every compactly supported distribution $\phi$ on $\mathbb{R}^{s}$.

If $\phi$ is a compactly supported continuous function on $\mathbb{R}^{s}$, and if $\phi$ satisfies condition (d), then it was proved in [14] that

$$
\phi *^{\prime} p=\hat{\phi}(-i D) p \quad \forall p \in \Pi_{k-1},
$$

where $i$ is the imaginary unit and $\hat{\phi}(-i D)$ denotes the differential operator given by the formal power series

$$
\sum_{\mu \geq 0} \frac{D^{\mu} \hat{\phi}(0)}{\mu !}(-i D)^{\mu} .
$$

For a given polynomial $p, D^{\mu} p=0$ if $|\mu|$ is sufficiently large. Thus, $\hat{\phi}(-i D)$ is well defined on $\Pi$. We indicate that (2.1) is also valid for a compactly supported distribution $\phi$ on $\mathbb{R}^{s}$ satisfying condition (d). To see this, choose a function $\rho \in$ $C_{c}^{\infty}\left(\mathbb{R}^{s}\right)$ such that $\hat{\rho}(0)=1$ and $D^{\nu} \hat{\rho}(0)=0$ for all $\nu$ with $0<|\nu| \leq k-1$. Let $\rho_{n}:=\rho(\cdot / n) / n^{s}$ for $n=1,2, \ldots$ Then for each $n, \phi_{n}:=\phi * \rho_{n}$, the convolution of $\phi$ with $\rho_{n}$, is a function in $C_{c}^{\infty}\left(\mathbb{R}^{s}\right)$. Moreover, the sequence $\left(\phi_{n}\right)_{n=1,2, \ldots}$ converges to $\phi$ in the sense that

$$
\lim _{n \rightarrow \infty}\left\langle\phi_{n}, f\right\rangle=\langle\phi, f\rangle \quad \forall f \in C_{c}^{\infty}\left(\mathbb{R}^{s}\right) .
$$


See $[1$, p. 97$]$ for these facts. Thus, we have $\hat{\phi}_{n}(\xi)=\hat{\phi}(\xi) \hat{\rho}_{n}(\xi)$ for $\xi \in \mathbb{R}^{s}$. Since $\phi$ satisfies condition (d), by using the Leibniz formula for differentiation, we get $D^{\mu} \hat{\phi}_{n}(2 \pi \beta)=0$ for $|\mu| \leq k-1$ and $\beta \in \mathbb{Z}^{s} \backslash\{0\}$. Hence (2.1) is applicable to $\phi_{n}$ and

$$
\phi_{n} *^{\prime} p=\hat{\phi}_{n}(-i D) p \quad \forall p \in \Pi_{k-1} .
$$

Letting $n \rightarrow \infty$ in the above equation, we obtain $\phi *^{\prime} p=\hat{\phi}(-i D) p$ for all $p \in \Pi_{k-1}$. Consequently, the linear mapping $\phi *^{\prime}$ given by $p \mapsto \phi *^{\prime} p$ maps $\Pi_{k-1}$ to $\Pi_{k-1}$. If, in addition, $\hat{\phi}(0) \neq 0$, then this mapping is one-to-one, and hence it is onto. This shows that $(\mathrm{d}) \Rightarrow(\mathrm{c})$ is valid for every compactly supported distribution $\phi$ on $\mathbb{R}^{s}$ with $\hat{\phi}(0) \neq 0$.

Next, we show that (c) $\Rightarrow$ (d) for every compactly supported distribution $\phi$ on $\mathbb{R}^{s}$. If $\phi$ is a compactly supported continuous function on $\mathbb{R}^{s}$, this was proved in [2] and [14]. Let $\phi$ be a compactly supported distribution on $\mathbb{R}^{s}$. For a fixed element $\beta \in \mathbb{Z}^{s} \backslash\{0\}$, choose a function $\rho \in C_{c}^{\infty}\left(\mathbb{R}^{s}\right)$ such that $\hat{\rho}(0) \neq 0$ and $\hat{\rho}(2 \pi \beta) \neq 0$. Then the convolution $\phi * \rho$ is a function in $C_{c}^{\infty}\left(\mathbb{R}^{s}\right)$ and its Fourier transform is $\hat{\phi} \hat{\rho}$. Note that the mapping $\rho *$ given by $q \mapsto \rho * q$ maps $\Pi_{k-1}$ to $\Pi_{k-1}$. Since $\hat{\rho}(0) \neq 0$, this mapping is one-to-one; hence it is onto. Thus, for $p \in \Pi_{k-1}$, we can find $q \in \Pi_{k-1}$ such that $p=\rho * q$. Since $\mathbb{S}(\phi) \supset \Pi_{k-1}$, there exists some $b \in \ell\left(\mathbb{Z}^{s}\right)$ such that $q=\phi *^{\prime} b$. It follows that $p=\rho *\left(\phi *^{\prime} b\right)=(\rho * \phi) *^{\prime} b$. This shows that $\mathbb{S}(\phi * \rho) \supset \Pi_{k-1}$. By what has been proved, $D^{\mu}(\hat{\phi} \hat{\rho})(2 \pi \beta)=0$ for all $\mu$ with $|\mu| \leq k-1$. Since $\hat{\rho}(2 \pi \beta) \neq 0$, we can write $\hat{\phi}=(\hat{\phi} \hat{\rho})(1 / \hat{\rho})$ in a neighborhood of $2 \pi \beta$. By applying the Leibniz formula for differentiation to this equation, we obtain $D^{\mu} \hat{\phi}(2 \pi \beta)=0$ for $|\mu| \leq k-1$. This shows that (c) $\Rightarrow$ (d) for every compactly supported distribution $\phi$ on $\mathbb{R}^{s}$.

To summarize, a compactly supported distribution $\phi$ on $\mathbb{R}^{s}$ with $\hat{\phi}(0) \neq 0$ possesses accuracy $k$ if and only if $D^{\mu} \hat{\phi}(2 \pi \beta)=0$ for all $\mu$ with $|\mu| \leq k-1$ and all $\beta \in \mathbb{Z}^{s} \backslash\{0\}$.

\section{Characterization of aCCURACy}

The purpose of this section is to give a characterization for the accuracy of a refinable function in terms of the refinement mask.

For an $s \times s$ dilation matrix $M$, let $\Gamma$ be a complete set of representatives of the distinct cosets of $\mathbb{Z}^{s} / M \mathbb{Z}^{s}$, and let $\Omega$ be a complete set of representatives of the distinct cosets of $\mathbb{Z}^{s} / M^{T} \mathbb{Z}^{s}$, where $M^{T}$ denotes the transpose of $M$. Evidently, $\# \Gamma=\# \Omega=|\operatorname{det} M|$. Without loss of any generality, we may assume that $0 \in \Gamma$ and $0 \in \Omega$.

Suppose $a$ is a finitely supported sequence on $\mathbb{Z}^{s}$ satisfying (1.2). Let $\phi$ be the normalized solution of the refinement equation (1.1). Taking Fourier transform of both sides of (1.1), we obtain

$$
\hat{\phi}(\xi)=H\left(\left(M^{T}\right)^{-1} \xi\right) \hat{\phi}\left(\left(M^{T}\right)^{-1} \xi\right), \quad \xi \in \mathbb{R}^{s},
$$

where

$$
H(\xi):=\sum_{\alpha \in \mathbb{Z}^{s}} a(\alpha) e^{-i \alpha \cdot \xi} / m, \quad \xi \in \mathbb{R}^{s} .
$$

Note that $H$ is a $2 \pi$-periodic function and $H(0)=1$. 
For a compactly supported distribution $\phi$ on $\mathbb{R}^{s}$, define

$$
N(\phi):=\left\{\xi \in \mathbb{R}^{s}: \hat{\phi}(\xi+2 \pi \beta)=0 \forall \beta \in \mathbb{Z}^{s}\right\} .
$$

If $\phi$ is a compactly supported function in $L_{p}\left(\mathbb{R}^{s}\right)(1 \leq p \leq \infty)$, then the shifts of $\phi$ are stable if and only if $N(\phi)$ is the empty set (see [19]).

Theorem 3.1. Let a be a finitely supported sequence on $\mathbb{Z}^{s}$ satisfying (1.2), and let $H$ be the function given in (3.2). If

$$
D^{\mu} H\left(2 \pi\left(M^{T}\right)^{-1} \omega\right)=0 \quad \forall \omega \in \Omega \backslash\{0\} \text { and }|\mu| \leq k-1,
$$

then the normalized solution $\phi$ of the refinement equation (1.1) has accuracy $k$. Conversely, if $\phi$ has accuracy $k$, and if $N(\phi) \cap\left(2 \pi\left(M^{T}\right)^{-1} \Omega\right)=\emptyset$, then (3.3) holds true.

Proof. Suppose that (3.3) is satisfied. Since $H$ is $2 \pi$-periodic, (3.3) implies

$$
D^{\mu} H\left(2 \pi\left(M^{T}\right)^{-1} \beta\right)=0 \quad \forall \beta \in \mathbb{Z}^{s} \backslash\left(M^{T} \mathbb{Z}^{s}\right) \text { and }|\mu| \leq k-1 .
$$

Let $f$ and $g$ be the functions given by

$$
f(\xi):=H\left(\left(M^{T}\right)^{-1} \xi\right) \quad \text { and } \quad g(\xi):=\hat{\phi}\left(\left(M^{T}\right)^{-1} \xi\right), \quad \xi \in \mathbb{R}^{s} .
$$

For $|\mu| \leq k-1$ and $\beta \in \mathbb{Z}^{s} \backslash\{0\}$, applying the Leibniz formula for differentiation to $(3.1)$, we obtain

$$
D^{\mu} \hat{\phi}(2 \pi \beta)=\sum_{\nu \leq \mu}\left(\begin{array}{l}
\mu \\
\nu
\end{array}\right) D^{\nu} f(2 \pi \beta) D^{\mu-\nu} g(2 \pi \beta) .
$$

By using the chain rule, we see that $D^{\nu} f(2 \pi \beta)$ is a linear combination of terms of the form $D^{\alpha} H\left(2 \pi\left(M^{T}\right)^{-1} \beta\right)$, where $\alpha \leq \nu$. In light of (3.4), these terms are equal to 0 if $\beta \in \mathbb{Z}^{s} \backslash\left(M^{T} \mathbb{Z}^{s}\right)$. This shows that $D^{\mu} \hat{\phi}(2 \pi \beta)=0$ for $\beta \in \mathbb{Z}^{s} \backslash\left(M^{T} \mathbb{Z}^{s}\right)$.

We shall prove that, for $r=0,1, \ldots, D^{\mu} \hat{\phi}(2 \pi \beta)=0$ for $\beta \in\left(\left(M^{T}\right)^{r} \mathbb{Z}^{s}\right) \backslash$ $\left(\left(M^{T}\right)^{r+1} \mathbb{Z}^{s}\right)$. This will be done by induction on $r$. The case $r=0$ was established above. Suppose $r \geq 1$ and our claim has been verified for $r-1$. Let $\beta \in\left(\left(M^{T}\right)^{r} \mathbb{Z}^{s}\right) \backslash$ $\left(\left(M^{T}\right)^{r+1} \mathbb{Z}^{s}\right)$. Then we have $\left(M^{T}\right)^{-1} \beta \in\left(\left(M^{T}\right)^{r-1} \mathbb{Z}^{s}\right) \backslash\left(\left(M^{T}\right)^{r} \mathbb{Z}^{s}\right)$. Hence, by the induction hypothesis, $D^{\mu} \hat{\phi}\left(2 \pi\left(M^{T}\right)^{-1} \beta\right)=0$ for $|\mu| \leq k-1$. Consequently, $D^{\mu} g(2 \pi \beta)=0$ for all $\mu$ with $|\mu| \leq k-1$. This in connection with (3.5) tells us that $D^{\mu} \hat{\phi}(2 \pi \beta)=0$ for $|\mu| \leq k-1$, thereby completing the induction procedure. The sufficiency part of the theorem has been established.

Conversely, suppose $\phi$ has accuracy $k$ and $N(\phi) \cap\left(2 \pi\left(M^{T}\right)^{-1} \Omega\right)=\emptyset$. Then

$$
D^{\mu} \hat{\phi}(2 \pi \beta)=0 \quad \forall \beta \in \mathbb{Z}^{s} \backslash\{0\} \text { and }|\mu| \leq k-1 .
$$

Let $\omega \in \Omega \backslash\{0\}$. Since $N(\phi) \cap\left(2 \pi\left(M^{T}\right)^{-1} \Omega\right)=\emptyset$, there exists some $\beta \in \mathbb{Z}^{s}$ such that $\hat{\phi}(\gamma) \neq 0$ for $\gamma:=2 \pi \beta+2 \pi\left(M^{T}\right)^{-1} \omega$. Thus, the following identity is valid for $\xi$ in a neighborhood of $\gamma$ :

$$
H(\xi)=\hat{\phi}\left(M^{T} \xi\right)[1 / \hat{\phi}(\xi)] .
$$

Let $h$ be the function given by $\xi \mapsto \hat{\phi}\left(M^{T} \xi\right), \xi \in \mathbb{R}^{s}$. By using the Leibniz formula for differentiation, we obtain

$$
D^{\mu} H(\gamma)=\sum_{\nu \leq \mu}\left(\begin{array}{l}
\mu \\
\nu
\end{array}\right) D^{\nu} h(\gamma) D^{\mu-\nu}[1 / \hat{\phi}](\gamma)
$$


By the chain rule, $D^{\nu} h(\gamma)$ is a linear combination of terms of the form $D^{\alpha} \hat{\phi}\left(M^{T} \gamma\right)$, where $\alpha \leq \nu$. Note that

$$
M^{T} \gamma=M^{T}\left(2 \pi \beta+2 \pi\left(M^{T}\right)^{-1} \omega\right)=2 \pi\left(M^{T}\right) \beta+2 \pi \omega \in 2 \pi \mathbb{Z}^{s} \backslash\{0\} .
$$

Hence $D^{\alpha} \hat{\phi}\left(M^{T} \gamma\right)=0$ for $|\alpha| \leq k-1$, because $\phi$ has accuracy $k$. Therefore we obtain $D^{\mu} H\left(2 \pi \beta+2 \pi\left(M^{T}\right)^{-1} \omega\right)=0$ for $|\mu| \leq k-1$. But $H$ is $2 \pi$-periodic. This shows that $D^{\mu} H\left(2 \pi\left(M^{T}\right)^{-1} \omega\right)=0$ for all $\omega \in \Omega \backslash\{0\}$ and $|\mu| \leq k-1$, as desired. The proof of the theorem is complete.

In the rest of this section we shall show that (3.3) is equivalent to saying that, for all $p \in \Pi_{k-1}$,

$$
\sum_{\beta \in \mathbb{Z}^{s}} a(M \beta) p(M \beta)=\sum_{\beta \in \mathbb{Z}^{s}} a(M \beta+\gamma) p(M \beta+\gamma) \quad \forall \gamma \in \Gamma .
$$

For this purpose, we first establish the following lemma.

Lemma 3.2. The matrix

$$
\frac{1}{\sqrt{m}}\left(e^{i 2 \pi M^{-1} \gamma \cdot \omega}\right)_{\gamma \in \Gamma, \omega \in \Omega}
$$

is a unitary one.

Proof. Let $\gamma \in \Gamma \backslash\{0\}$. We claim that there exists some $\omega^{\prime} \in \Omega$ such that $M^{-1} \gamma \cdot \omega^{\prime} \notin$ $\mathbb{Z}$. Any element $\beta \in \mathbb{Z}^{s}$ can be represented as $M^{T} \alpha+\omega$ for some $\alpha \in \mathbb{Z}^{s}$ and $\omega \in \Omega$. Note that $\left(M^{-1} \gamma\right) \cdot\left(M^{T} \alpha\right)=\gamma \cdot \alpha \in \mathbb{Z}$ for all $\alpha \in \mathbb{Z}^{s}$. Hence $M^{-1} \gamma \cdot \omega^{\prime} \in \mathbb{Z}$ for all $\omega^{\prime} \in \Omega$ implies that $M^{-1} \gamma \cdot \beta \in \mathbb{Z}$ for all $\beta \in \mathbb{Z}^{s}$. In other words, $M^{-1} \gamma \in \mathbb{Z}^{s}$, and hence $\gamma \in M \mathbb{Z}^{s}$, which contradicts the assumption $\gamma \in \Gamma \backslash\{0\}$. This verifies our claim.

For a fixed element $\gamma$ in $\Gamma \backslash\{0\}$, let

$$
\sigma:=\sum_{\omega \in \Omega} e^{i 2 \pi M^{-1} \gamma \cdot \omega} .
$$

Choose $\omega^{\prime} \in \Omega$ such that $M^{-1} \gamma \cdot \omega^{\prime} \notin \mathbb{Z}$. We have

$$
e^{i 2 \pi M^{-1} \gamma \cdot \omega^{\prime}} \sigma=\sum_{\omega \in \Omega} e^{i 2 \pi\left(M^{-1} \gamma\right) \cdot\left(\omega+\omega^{\prime}\right)}=\sum_{\omega \in \Omega} e^{i 2 \pi M^{-1} \gamma \cdot \omega}=\sigma .
$$

Since $e^{i 2 \pi M^{-1} \gamma \cdot \omega^{\prime}} \neq 1$, it follows that $\sigma=0$. This shows that

$$
\sum_{\omega \in \Omega} e^{i 2 \pi M^{-1} \gamma \cdot \omega}=0 \quad \forall \gamma \in \Gamma \backslash\{0\} .
$$

Similarly, we can prove that

$$
\sum_{\gamma \in \Gamma} e^{i 2 \pi M^{-1} \gamma \cdot \omega}=0 \quad \forall \omega \in \Omega \backslash\{0\} .
$$

Finally, the matrix in (3.7) is unitary if and only if for every pair of elements $\gamma, \gamma^{\prime} \in \Gamma$,

$$
\frac{1}{m} \sum_{\omega \in \Omega} e^{i 2 \pi M^{-1}\left(\gamma-\gamma^{\prime}\right) \cdot \omega}= \begin{cases}1 & \text { if } \gamma=\gamma^{\prime} \\ 0 & \text { if } \gamma \neq \gamma^{\prime}\end{cases}
$$

For $\gamma=\gamma^{\prime}$, this comes from the fact $\# \Omega=m$; for $\gamma \neq \gamma^{\prime}$, this follows from (3.8). 
Lemma 3.3. Let a be a finitely supported sequence satisfying (1.2), and let $H$ be the function given in (3.2). Then the following two conditions are equivalent for every polynomial $p$ :

(a) $p(i D) H\left(2 \pi\left(M^{T}\right)^{-1} \omega\right)=0$ for all $\omega \in \Omega \backslash\{0\}$.

(b) $\sum_{\beta \in \mathbb{Z}^{s}} a(M \beta) p(M \beta)=\sum_{\beta \in \mathbb{Z}^{s}} a(M \beta+\gamma) p(M \beta+\gamma)$ for all $\gamma \in \Gamma$.

Proof. By (3.2) we have

$$
m p(i D) H(\xi)=\sum_{\alpha \in \mathbb{Z}^{s}} a(\alpha) p(\alpha) e^{-i \alpha \cdot \xi}, \quad \xi \in \mathbb{R}^{s} .
$$

An element $\alpha \in \mathbb{Z}^{s}$ can be written uniquely as $M \beta+\gamma$ with $\beta \in \mathbb{Z}^{s}$ and $\gamma \in \Gamma$. Observe that, for $\xi:=2 \pi\left(M^{T}\right)^{-1} \omega$,

$$
-i \alpha \cdot \xi=-i(M \beta+\gamma) \cdot 2 \pi\left(M^{T}\right)^{-1} \omega=-i 2 \pi \beta \cdot \omega-i 2 \pi \gamma \cdot\left(M^{T}\right)^{-1} \omega .
$$

Hence we have

$$
m p(i D) H\left(2 \pi\left(M^{T}\right)^{-1} \omega\right)=\sum_{\gamma \in \Gamma} b(\gamma) e^{-i 2 \pi \gamma \cdot\left(M^{T}\right)^{-1} \omega},
$$

where

$$
b(\gamma):=\sum_{\beta \in \mathbb{Z}^{s}} a(M \beta+\gamma) p(M \beta+\gamma) .
$$

Condition (b) says that $b(\gamma)=b(0)$ for all $\gamma \in \Gamma$. Hence by (3.9) we deduce from (3.10) that

$$
m p(i D) H\left(2 \pi\left(M^{T}\right)^{-1} \omega\right)=b(0) \sum_{\gamma \in \Gamma} e^{-i 2 \pi \gamma \cdot\left(M^{T}\right)^{-1} \omega}=0
$$

for all $\omega \in \Omega \backslash\{0\}$. This shows that (b) $\Rightarrow$ (a).

Conversely, (3.10) tells us that condition (a) implies

$$
\sum_{\gamma \in \Gamma} b(\gamma) e^{-i 2 \pi M^{-1} \gamma \cdot \omega}=0 \quad \forall \omega \in \Omega \backslash\{0\} .
$$

Let $\eta$ be an element of $\Gamma$. Then it follows that

$$
\sum_{\omega \in \Omega} e^{i 2 \pi M^{-1} \eta \cdot \omega} \sum_{\gamma \in \Gamma} b(\gamma) e^{-i 2 \pi M^{-1} \gamma \cdot \omega}=\sum_{\gamma \in \Gamma} b(\gamma) .
$$

On the other hand,

$$
\sum_{\omega \in \Omega} e^{i 2 \pi M^{-1} \eta \cdot \omega} \sum_{\gamma \in \Gamma} b(\gamma) e^{-i 2 \pi M^{-1} \gamma \cdot \omega}=\sum_{\gamma \in \Gamma} b(\gamma) \sum_{\omega \in \Omega} e^{i 2 \pi M^{-1}(\eta-\gamma) \cdot \omega}=m b(\eta),
$$

since $\sum_{\omega \in \Omega} e^{i 2 \pi M^{-1}(\eta-\gamma) \cdot \omega}=0$ for $\gamma \neq \eta$, by Lemma 3.2. This shows $m b(\eta)=$ $\sum_{\gamma \in \Gamma} b(\gamma)$. Therefore $b(\eta)=b(0)$ for all $\eta \in \Gamma$. In other words, (a) implies (b).

If an element $a \in \ell_{0}\left(\mathbb{Z}^{s}\right)$ satisfies (3.6) for all $p \in \Pi_{k-1}$, then we say that $a$ satisfies the sum rules of order $k$. The results of this section can be summarized as follows: If the refinement mask $a$ satisfies the sum rules of order $k$, then the normalized solution $\phi$ of the refinement equation with mask $a$ has accuracy $k$. Conversely, if $\phi$ has accuracy $k$, and if $N(\phi) \cap\left(2 \pi\left(M^{T}\right)^{-1} \Omega\right)=\emptyset$, then $a$ satisfies the sum rules of order $k$. 


\section{EXAMPLES}

In this section we give several examples to illustrate the general theory.

The symbol of a sequence $a \in \ell_{0}\left(\mathbb{Z}^{s}\right)$ is the Laurent polynomial $\tilde{a}(z)$ given by

$$
\tilde{a}(z):=\sum_{\alpha \in \mathbb{Z}^{s}} a(\alpha) z^{\alpha}, \quad z \in(\mathbb{C} \backslash\{0\})^{s},
$$

where $z^{\alpha}:=z_{1}^{\alpha_{1}} \cdots z_{s}^{\alpha_{s}}$ for $z=\left(z_{1}, \ldots, z_{s}\right) \in \mathbb{C}^{s}$ and $\alpha=\left(\alpha_{1}, \ldots, \alpha_{s}\right) \in \mathbb{Z}^{s}$. If $a$ is supported on $[0, N]^{s}$ for some positive integer $N$, then $\tilde{a}(z)$ is a polynomial of $z$.

In the univariate case $(s=1)$, if $a$ satisfies the sum rules of order $k$, then $\tilde{a}(z)$ is divisible by $(1+z)^{k}$ (see, e.g., [8]). In the multivariate case $(s>1)$, this is no longer true.

Example 4.1. Let $s=2$ and $M=2 I$, where $I$ is the $2 \times 2$ identity matrix. Let $a$ be the sequence on $\mathbb{Z}^{2}$ given by its symbol

$$
\tilde{a}(z):=z_{1}^{2}+z_{2}+z_{1} z_{2}+z_{1} z_{2}^{2} .
$$

Then $a$ satisfies the sum rules of order 1 . But the polynomial $\tilde{a}(z)$ is irreducible.

It is easy to verify that $a$ satisfies the sum rules of order 1 . Let us show that $\tilde{a}(z)$ is irreducible. Suppose to the contrary that $\tilde{a}(z)$ is reducible. Then $\tilde{a}(z)$ can be factored as

$$
\tilde{a}(z)=f(z) g(z)
$$

where $f$ and $g$ are polynomials of (total) degree at least 1 . Since the degree of $\tilde{a}(z)$ is 3 , the degree of either $f$ or $g$ is 1 . Suppose the degree of $f$ is 1 and

$$
f\left(z_{1}, z_{2}\right)=\lambda z_{1}+\mu z_{2}+\nu,
$$

where $\lambda, \mu, \nu$ are complex numbers and either $\lambda \neq 0$ or $\mu \neq 0$. If $\lambda \neq 0$, then for all $z_{2} \in \mathbb{C}, f\left(-\left(\mu z_{2}+\nu\right) / \lambda, z_{2}\right)=0$, and so

$$
\tilde{a}\left(-\left(\mu z_{2}+\nu\right) / \lambda, z_{2}\right)=0 \quad \forall z_{2} \in \mathbb{C} .
$$

If $\mu \neq 0$, then $\tilde{a}\left(-\left(\mu z_{2}+\nu\right) / \lambda, z_{2}\right)$ is a polynomial of $z_{2}$ of degree 3 with $-\mu / \lambda$ being its leading coefficient. Hence $\mu=0$. But it is also impossible that $\tilde{a}\left(-\nu / \lambda, z_{2}\right)=0$ for all $z_{2} \in \mathbb{C}$. Thus, we must have $\lambda=0$, and hence $\tilde{a}\left(z_{1},-\nu / \mu\right)=0$ for all $z_{1} \in \mathbb{C}$. However, $\tilde{a}\left(z_{1},-\nu / \mu\right)$ is a polynomial of $z_{1}$ of degree 2 with 1 being its leading coefficient. This contradiction shows that $\tilde{a}(z)$ is irreducible.

Let $a$ be the sequence given as above, and let $\phi$ be the normalized solution of the refinement equation

$$
\phi=\sum_{\alpha \in \mathbb{Z}^{2}} a(\alpha) \phi(2 \cdot-\alpha)
$$

Then $\phi$ lies in $L_{2}\left(\mathbb{R}^{2}\right)$. This can be verified by using the results in [12]. Let $b$ be the element in $\ell_{0}\left(\mathbb{Z}^{2}\right)$ given by its symbol

$$
\tilde{b}(z):=|\tilde{a}(z)|^{2} / 4 \quad \text { for }\left|z_{1}\right|=1 \text { and }\left|z_{2}\right|=1 .
$$

We have

$$
\begin{aligned}
4 \tilde{b}(z)=4 & +z_{1}+z_{1}^{-1}+z_{2}+z_{2}^{-1}+z_{1} z_{2}+z_{1}^{-1} z_{2}^{-1} \\
& +z_{1} z_{2}^{-1}+z_{1}^{-1} z_{2}+z_{1} z_{2}^{-2}+z_{1}^{-1} z_{2}^{2}+z_{1}^{2} z_{2}^{-1}+z_{1}^{-2} z_{2} .
\end{aligned}
$$


Let $B$ be the linear operator on $\ell_{0}\left(\mathbb{Z}^{2}\right)$ given by

$$
B v(\alpha):=\sum_{\beta \in \mathbb{Z}^{2}} b(2 \alpha-\beta) v(\beta), \quad \alpha \in \mathbb{Z}^{2},
$$

where $v \in \ell_{0}\left(\mathbb{Z}^{2}\right)$. Let $W$ be the $B$-invariant subspace generated by $-\delta_{-e_{1}}+2 \delta-\delta_{e_{1}}$ and $-\delta_{-e_{2}}+2 \delta-\delta_{e_{2}}$. Then the spectral radius $\rho$ of the linear operator $\left.B\right|_{W}$ is $3 / 4$. Since $\rho<1$, by [12, Theorems 3.3 and 4.1], the subdivision scheme associated with $a$ is $L_{2}$-convergent. Therefore, $\phi \in L_{2}\left(\mathbb{R}^{2}\right)$ and the shifts of $\phi$ are orthonormal (see [11]). We conclude that the optimal order of approximation provided by $\mathbb{S}(\phi)$ is 1 .

If the refinement mask $a$ satisfies the sum rules of order $k$, then the normalized solution $\phi$ of the refinement equation with mask $a$ has accuracy $k$. However, if the condition $N(\phi) \cap\left(2 \pi\left(M^{T}\right)^{-1} \Omega\right)=\emptyset$ is not satisfied, then $\phi$ could have higher accuracy. For instance, the function $\phi$ on $\mathbb{R}$ given by $\phi(x)=1 / 2$ for $0 \leq x<2$ and $\phi(x)=0$ for $x \in \mathbb{R} \backslash[0,2)$ satisfies the refinement equation

$$
\phi=\sum_{\alpha \in \mathbb{Z}} a(\alpha) \phi(2 \cdot-\alpha)
$$

where the symbol of the mask $a$ is $\tilde{a}(z)=1+z^{2}$. Then $a$ does not satisfy the sum rules of order 1 . But $\phi$ has accuracy 1 , and $\mathbb{S}(\phi)$ provides $L_{\infty}$-approximation order 1. The following is an example in the two-dimensional case.

Example 4.2. Let $\phi$ be the Zwart-Powell element defined by its Fourier transform

$$
\hat{\phi}\left(\xi_{1}, \xi_{2}\right):=g\left(\xi_{1}\right) g\left(\xi_{2}\right) g\left(\xi_{1}+\xi_{2}\right) g\left(-\xi_{1}+\xi_{2}\right), \quad\left(\xi_{1}, \xi_{2}\right) \in \mathbb{R}^{2},
$$

where $g$ is the function on $\mathbb{R}$ given by $\xi \mapsto\left(1-e^{-i \xi}\right) /(i \xi), \xi \in \mathbb{R}$. Then $\phi$ is a compactly supported continuous function on $\mathbb{R}^{2}$ and $\mathbb{S}(\phi)$ provides $L_{\infty}$-approximation order 3. On the other hand, $\phi$ is refinable but the corresponding mask does not satisfy the sum rules of order 3 .

For the first statement the reader is referred to [5, p. 72]. Let us verify the second statement. From [5, p. 140] we know that the Zwart-Powell element $\phi$ is refinable and the corresponding mask $a$ is given by $a(\alpha)=0$ for $\alpha \in \mathbb{Z}^{2} \backslash[-1,2] \times[0,3]$ and

$$
\left(a\left(\alpha_{1}, \alpha_{2}\right)\right)_{-1 \leq \alpha_{1} \leq 2,0 \leq \alpha_{2} \leq 3}=\frac{1}{4}\left[\begin{array}{cccc}
0 & 1 & 1 & 0 \\
1 & 2 & 2 & 1 \\
1 & 2 & 2 & 1 \\
0 & 1 & 1 & 0
\end{array}\right] .
$$

Evidently, the mask $a$ satisfies the sum rules of order 2, but $a$ does not satisfy the sum rules of order 3 . Note that $(\pi, \pi) \in N(\phi)$ in this case.

Example 4.3. Let $M$ be the matrix

$$
\left(\begin{array}{rr}
1 & -1 \\
1 & 1
\end{array}\right),
$$

and let $a$ be the sequence on $\mathbb{Z}^{2}$ such that $a(\alpha)=0$ for $\alpha \in \mathbb{Z}^{2} \backslash[-2,2]^{2}$ and

$$
\left(a\left(\alpha_{1}, \alpha_{2}\right)\right)_{-2 \leq \alpha_{1}, \alpha_{2} \leq 2}=\frac{1}{32}\left[\begin{array}{rrrrr}
0 & -1 & 0 & -1 & 0 \\
-1 & 0 & 10 & 0 & -1 \\
0 & 10 & 32 & 10 & 0 \\
-1 & 0 & 10 & 0 & -1 \\
0 & -1 & 0 & -1 & 0
\end{array}\right] .
$$


Let $\phi$ be the normalized solution of the refinement equation (1.1) with mask $a$ and dilation matrix $M$ given as above. Then $\phi$ is a compactly supported continuous function on $\mathbb{R}^{2}$, and the optimal approximation order provided by $\mathbb{S}(\phi)$ is 4 .

Let us verify that $a$ satisfies the sum rules of order 4 . We observe that $\alpha=$ $\left(\alpha_{1}, \alpha_{2}\right)$ lies in $M \mathbb{Z}^{2}$ if and only if $\alpha_{1}+\alpha_{2}$ is an even integer. Hence the sum rule for a polynomial $p$ of two variables reads as follows:

$$
\sum_{\alpha_{1}+\alpha_{2} \in 2 \mathbb{Z}} p(\alpha) a(\alpha)=\sum_{\beta_{1}+\beta_{2} \notin 2 \mathbb{Z}} p(\beta) a(\beta),
$$

that is,

$$
32 p(0,0)=10 \sum_{\left|\alpha_{1}\right|+\left|\alpha_{2}\right|=1} p\left(\alpha_{1}, \alpha_{2}\right)-\sum_{\left|\alpha_{1}\right|+\left|\alpha_{2}\right|=3} p\left(\alpha_{1}, \alpha_{2}\right) .
$$

We can easily verify that this condition is satisfied for all $p \in \Pi_{3}$, but it is not satisfied for the monomial $p$ given by $p\left(x_{1}, x_{2}\right)=x_{1}^{2} x_{2}^{2},\left(x_{1}, x_{2}\right) \in \mathbb{R}^{2}$. Therefore the refinement mask $a$ satisfies the sum rules of order 4 , but not of order 5 .

In the present case, $\Omega:=\{(0,0),(1,0)\}$ is a complete set of representatives of the distinct cosets of $\mathbb{Z}^{2} / M^{T} \mathbb{Z}^{2}$. We have $2 \pi\left(M^{T}\right)^{-1} \Omega=\{(0,0),(\pi, \pi)\}$. Since $\hat{\phi}(0,0)=1$, in order to verify the condition $N(\phi) \cap\left(2 \pi\left(M^{T}\right)^{-1} \Omega\right)=\emptyset$, it suffices to show that $\hat{\phi}(\pi, \pi) \neq 0$. For this purpose, we observe that

$$
\hat{\phi}(\xi)=\prod_{k=1}^{\infty} H\left(\left(M^{T}\right)^{-k} \xi\right), \quad \xi \in \mathbb{R}^{2},
$$

where

$$
\begin{array}{r}
H(\xi)=\left[32+20\left(\cos \xi_{1}+\cos \xi_{2}\right)-4 \cos \left(2 \xi_{1}+\xi_{2}\right)-4 \cos \left(\xi_{1}+2 \xi_{2}\right)\right] / 64, \\
\xi=\left(\xi_{1}, \xi_{2}\right) \in \mathbb{R}^{2} .
\end{array}
$$

We have $\left(M^{T}\right)^{-1}(\pi, \pi)^{T}=(0, \pi)^{T}$ and $H(0, \pi)>0$. Suppose

$$
\left(\eta_{1}, \eta_{2}\right)^{T}=\left(M^{T}\right)^{-k}(\pi, \pi)^{T}
$$

for some integer $k \geq 2$. Then $\left|\eta_{1}\right| \leq \pi / 2$ and $\left|\eta_{2}\right| \leq \pi / 2$, so $H\left(\eta_{1}, \eta_{2}\right)>0$. It follows that $\hat{\phi}(\pi, \pi) \neq 0$. Consequently, the exact accuracy of $\phi$ is 4 .

By using the methods in [12], we can easily prove that the subdivision scheme associated with mask $a$ and dilation matrix $M$ converges uniformly. Consequently, $\phi$ is a continuous function. We conclude that the optimal approximation order provided by $\mathbb{S}(\phi)$ is 4 .

\section{The SUBDIVISION AND TRANSITION OPERATORS}

We introduce two linear operators associated with a refinement equation. One is the subdivision operator, and the other is the transition operator. When the dilation matrix $M$ is 2 times the identity matrix, the spectral properties of the subdivision and transition operators were studied in [10] and [18]. In this section, we extend the study to the case in which $M$ is a general dilation matrix.

Let $X$ and $Y$ be two linear spaces, and $T$ a linear mapping from $X$ to $Y$. The kernel of $T$, denoted by $\operatorname{ker}(T)$, is the subspace of $X$ consisting of all $x \in X$ such that $T x=0$. 
Let $a$ be an element in $\ell_{0}\left(\mathbb{Z}^{s}\right)$ and let $M$ be a dilation matrix. The subdivision operator $S_{a}$ is the linear operator on $\ell\left(\mathbb{Z}^{s}\right)$ defined by

$$
S_{a} u(\alpha):=\sum_{\beta \in \mathbb{Z}^{s}} a(\alpha-M \beta) u(\beta), \quad \alpha \in \mathbb{Z}^{s},
$$

where $u \in \ell\left(\mathbb{Z}^{s}\right)$. The transition operator $T_{a}$ is the linear operator on $\ell_{0}\left(\mathbb{Z}^{s}\right)$ defined by

$$
T_{a} v(\alpha):=\sum_{\beta \in \mathbb{Z}^{s}} a(M \alpha-\beta) v(\beta), \quad \alpha \in \mathbb{Z}^{s},
$$

where $v \in \ell_{0}\left(\mathbb{Z}^{s}\right)$.

The following theorem shows that the subdivision operator $S_{a}$ and the transition operator $T_{a}$ have the same nonzero eigenvalues. We use $I$ and $I_{0}$ to denote the identity mapping on $\ell\left(\mathbb{Z}^{s}\right)$ and $\ell_{0}\left(\mathbb{Z}^{s}\right)$, respectively.

Theorem 5.1. The transition operator $T_{a}$ has only finitely many nonzero eigenvalues. For $\sigma \in \mathbb{C} \backslash\{0\}$, the linear spaces $\operatorname{ker}\left(S_{a}-\sigma I\right)$ and $\operatorname{ker}\left(T_{a}-\sigma I_{0}\right)$ have the same dimension. In particular, $\sigma$ is an eigenvalue of $S_{a}$ if and only if it is an eigenvalue of $T_{a}$.

Proof. For $N=1,2, \ldots$, let $E_{N}$ denote the cube $[-N, N]^{s}$. Choose $N$ such that $E_{N-1}$ contains $\operatorname{supp} a:=\left\{\alpha \in \mathbb{Z}^{s}: a(\alpha) \neq 0\right\}$. Let $K:=\sum_{n=1}^{\infty} M^{-n} E_{N}$. In other words, $x$ belongs to $K$ if and only if $x=\sum_{n=1}^{\infty} M^{-n} y_{n}$ for some sequence of elements $y_{n} \in E_{N}$. Let $\ell(K)$ denote the linear space of all (finite) sequences on $K \cap \mathbb{Z}^{s}$. Consider the linear mapping $A$ on $\ell(K)$ given by

$$
A v(\alpha):=\sum_{\beta \in K \cap \mathbb{Z}^{s}} a(M \alpha-\beta) v(\beta), \quad \alpha \in K \cap \mathbb{Z}^{s},
$$

where $v \in \ell(K)$. The dual mapping $A^{\prime}$ of $A$ is given by

$$
A^{\prime} u(\beta):=\sum_{\alpha \in K \cap \mathbb{Z}^{s}} u(\alpha) a(M \alpha-\beta), \quad \beta \in K \cap \mathbb{Z}^{s},
$$

where $u \in \ell(K)$. Let $I_{K}$ denote the identity mapping on $\ell(K)$. Since $\ell(K)$ is finite dimensional, we have

$$
\operatorname{dim}\left(\operatorname{ker}\left(A-\sigma I_{K}\right)\right)=\operatorname{dim}\left(\operatorname{ker}\left(A^{\prime}-\sigma I_{K}\right)\right) .
$$

Thus, in order to establish the theorem, it suffices to prove the following two relations:

$$
\operatorname{dim}\left(\operatorname{ker}\left(T_{a}-\sigma I_{0}\right)\right)=\operatorname{dim}\left(\operatorname{ker}\left(A-\sigma I_{K}\right)\right)
$$

and

$$
\operatorname{dim}\left(\operatorname{ker}\left(S_{a}-\sigma I\right)\right)=\operatorname{dim}\left(\operatorname{ker}\left(A^{\prime}-\sigma I_{K}\right)\right)
$$

For this purpose, we introduce the sets $K_{j}(j=0,1, \ldots)$ as follows:

$$
K_{j}:=M^{j-1} E_{1}+\cdots+E_{1}+K .
$$

In particular, $K_{0}=K$. Evidently, $K_{j} \subseteq K_{j+1}$ for $j=0,1, \ldots$, and $\mathbb{R}^{s}=\bigcup_{j=0}^{\infty} K_{j}$. Moreover,

$$
M^{-1}\left(K_{j}+\operatorname{supp} a\right) \subseteq K_{j-1}, \quad j=1,2, \ldots
$$


Indeed, we have $M^{-1} K+M^{-1} E_{N}=K$, and hence

$$
\begin{aligned}
M^{-1}\left(K_{j}+\operatorname{supp} a\right) & \subseteq M^{j-2} E_{1}+\cdots+E_{1}+M^{-1} E_{1}+M^{-1} K+M^{-1} E_{N-1} \\
& \subseteq K_{j-1} .
\end{aligned}
$$

Suppose $\sigma \neq 0$ and $v \in \operatorname{ker}\left(T_{a}-\sigma I_{0}\right)$. Then supp $v \subseteq K_{j}$ for some $j \geq 1$. We observe that $T_{a} v(\alpha) \neq 0$ implies $M \alpha-\beta \in \operatorname{supp} a$ for some $\beta \in K_{j}$. It follows that $\alpha \in M^{-1}\left(\operatorname{supp} a+K_{j}\right) \subseteq K_{j-1}$, by (5.3). In other words, $\operatorname{supp}\left(T_{a} v\right) \subseteq K_{j-1}$. Using this relation repeatedly, we obtain $\operatorname{supp}\left(T_{a}^{j} v\right) \subseteq K$. But $v=T_{a} v / \sigma=\left(T_{a}^{j} v\right) / \sigma^{j}$. Therefore, $\operatorname{supp} v \subseteq K$, and $\left.v\right|_{K \cap \mathbb{Z}^{s}}$ belongs to $\operatorname{ker}\left(A-\sigma I_{K}\right)$. This shows that the restriction mapping $P:\left.v \mapsto v\right|_{K \cap \mathbb{Z}^{s}}$ maps $\operatorname{ker}\left(T_{a}-\sigma I_{0}\right)$ to $\operatorname{ker}\left(A-\sigma I_{K}\right)$. Moreover, $\left.v\right|_{K \cap \mathbb{Z}^{s}}=0$ implies $v=0$. So $P$ is one-to-one. Let us show that $P$ is also onto. Suppose $A w=\sigma w$ for some $w \in \ell(K)$. Define $v(\alpha):=w(\alpha)$ for $\alpha \in K \cap \mathbb{Z}^{s}$ and $v(\alpha):=0$ for $\alpha \in \mathbb{Z}^{s} \backslash K$. Then $T_{a} v=\sigma v$. Thus, $P$ is one-to-one and onto, thereby establishing (5.1).

In order to prove (5.2), we consider the mapping $Q:\left.u \mapsto u^{*}\right|_{K \cap \mathbb{Z}^{s}}$, where $u^{*}$ is the sequence given by $u^{*}(\alpha):=u(-\alpha), \alpha \in \mathbb{Z}^{s}$. Suppose $u \in \operatorname{ker}\left(S_{a}-\sigma I\right)$. Then

$$
u(\alpha)=\frac{1}{\sigma} \sum_{\beta \in \mathbb{Z}^{s}} a(\alpha-M \beta) u(\beta), \quad \alpha \in \mathbb{Z}^{s} .
$$

It follows that

$$
u^{*}(\alpha)=\frac{1}{\sigma} \sum_{\beta \in \mathbb{Z}^{s}} u^{*}(\beta) a(M \beta-\alpha), \quad \alpha \in \mathbb{Z}^{s} .
$$

For $\alpha \in K_{j}(j \geq 1), a(M \beta-\alpha) \neq 0$ only if $\beta \in M^{-1}\left(\operatorname{supp} a+K_{j}\right) \subseteq K_{j-1}$. Hence

$$
u^{*}(\alpha)=\frac{1}{\sigma} \sum_{\beta \in K_{j-1} \cap \mathbb{Z}^{s}} u^{*}(\beta) a(M \beta-\alpha) \quad \text { for } \alpha \in K_{j} \cap \mathbb{Z}^{s} .
$$

This shows that $\left.u^{*}\right|_{K \cap \mathbb{Z}^{s}}$ belongs to $\operatorname{ker}\left(A^{\prime}-\sigma I_{K}\right)$. Thus, $Q$ maps $\operatorname{ker}\left(S_{a}-\sigma I\right)$ to $\operatorname{ker}\left(A^{\prime}-\sigma I_{K}\right)$. Moreover, if $u^{*}(\alpha)=0$ for $\alpha \in K \cap \mathbb{Z}^{s}$, then it follows from (5.4) that $u^{*}(\alpha)=0$ for $\alpha \in K_{j} \cap \mathbb{Z}^{s}, j=1,2, \ldots$ But $\mathbb{R}^{s}=\bigcup_{j=1}^{\infty} K_{j}$; hence $u^{*}(\alpha)=0$ for all $\alpha \in \mathbb{Z}^{s}$. Thus, the mapping $Q$ is one-to-one. It is also onto. Indeed, if $w \in \operatorname{ker}\left(A^{\prime}-\sigma I_{K}\right)$, then

$$
w(\alpha)=\frac{1}{\sigma} \sum_{\beta \in K \cap \mathbb{Z}^{s}} w(\beta) a(M \beta-\alpha), \quad \alpha \in K \cap \mathbb{Z}^{s} .
$$

For $\alpha \in K \cap \mathbb{Z}^{s}$, let $u^{*}(\alpha):=w(\alpha)$; for $\alpha \in\left(K_{j} \backslash K_{j-1}\right) \cap \mathbb{Z}^{s}(j=1,2, \ldots)$, let $u^{*}(\alpha)$ be determined recursively by (5.4). Then $u \in \operatorname{ker}\left(S_{a}-\sigma I\right)$ and $Q u=w$. Thus, $Q$ is one-to-one and onto, so that (5.2) is valid. The proof of the theorem is complete.

A sequence $u$ on $\mathbb{Z}^{s}$ is called a polynomial sequence if there exists a polynomial $p$ such that $u(\alpha)=p(\alpha)$ for all $\alpha \in \mathbb{Z}^{s}$. The degree of $u$ is the same as the degree of $p$. For a nonnegative integer $k$, let $P_{k}$ be the linear space of all polynomial sequences of degree at most $k$, and let

$$
V_{k}:=\left\{v \in \ell_{0}\left(\mathbb{Z}^{s}\right): \sum_{\alpha \in \mathbb{Z}^{s}} p(\alpha) v(\alpha)=0 \forall p \in \Pi_{k}\right\} .
$$


For $u \in \ell\left(\mathbb{Z}^{s}\right)$ and $v \in \ell_{0}\left(\mathbb{Z}^{s}\right)$, we define

$$
\langle u, v\rangle:=\sum_{\alpha \in \mathbb{Z}^{s}} u(\alpha) v(\alpha) .
$$

Theorem 5.2. Let $M$ be an $s \times s$ dilation matrix and $\Omega$ a complete set of representatives of the distinct cosets of $\mathbb{Z}^{s} / M^{T} \mathbb{Z}^{s}$. For any $a \in \ell_{0}\left(\mathbb{Z}^{s}\right)$, the following statements are equivalent:

(a) The sequence a satisfies the sum rules of order $k+1$.

(b) $V_{k}$ is invariant under the transition operator $T_{a}$.

(c) $P_{k}$ is invariant under the subdivision operator $S_{a}$.

(d) $D^{\mu} H\left(2 \pi\left(M^{T}\right)^{-1} \omega\right)=0$ for all $|\mu| \leq k$ and all $\omega \in \Omega \backslash\{0\}$.

Proof. $(\mathrm{a}) \Rightarrow(\mathrm{b})$ : Let $p \in \Pi_{k}$ and $v \in V_{k}$. We have

$$
\sum_{\alpha \in \mathbb{Z}^{s}} p(\alpha) T_{a} v(\alpha)=\sum_{\beta \in \mathbb{Z}^{s}}\left[\sum_{\alpha \in \mathbb{Z}^{s}} p(\alpha) a(M \alpha-\beta)\right] v(\beta) .
$$

Let $q(x):=p\left(M^{-1} x\right), x \in \mathbb{R}^{s}$. Then $p(x)=q(M x), x \in \mathbb{R}^{s}$. By Taylor's formula, we have

$$
q(M \alpha)=q(M \alpha-\beta+\beta)=\sum_{|\mu| \leq k} q_{\mu}(M \alpha-\beta) \beta^{\mu},
$$

where $q_{\mu}:=D^{\mu} q / \mu ! \in \Pi_{k}$. Hence

$$
\sum_{\alpha \in \mathbb{Z}^{s}} p(\alpha) a(M \alpha-\beta)=\sum_{\alpha \in \mathbb{Z}^{s}} q(M \alpha) a(M \alpha-\beta)=\sum_{|\mu| \leq k} c_{\mu} \beta^{\mu},
$$

where

$$
c_{\mu}:=\sum_{\alpha \in \mathbb{Z}^{s}} q_{\mu}(M \alpha-\beta) a(M \alpha-\beta)
$$

is independent of $\beta$, by condition (a). Thus, we obtain

$$
\sum_{\alpha \in \mathbb{Z}^{s}} p(\alpha) T_{a} v(\alpha)=\sum_{|\mu| \leq k} c_{\mu} \sum_{\beta \in \mathbb{Z}^{s}} \beta^{\mu} v(\beta)=0
$$

because $v \in V_{k}$. This shows that $T_{a} v \in V_{k}$ for $v \in V_{k}$. In other words, $V_{k}$ is invariant under $T_{a}$.

(b) $\Rightarrow(\mathrm{c})$ : Suppose $p \in P_{k}$. We wish to show that $u:=S_{a} p$ lies in $P_{k}$. We claim that $\langle u, v\rangle=0$ for all $v \in V_{k}$. Indeed,

$$
\begin{aligned}
\langle u, v\rangle & =\sum_{\alpha \in \mathbb{Z}^{s}} u(\alpha) v(\alpha)=\sum_{\alpha \in \mathbb{Z}^{s}} \sum_{\beta \in \mathbb{Z}^{s}} a(\alpha-M \beta) p(\beta) v(\alpha) \\
& =\sum_{\beta \in \mathbb{Z}^{s}} p(-\beta) \sum_{\alpha \in \mathbb{Z}^{s}} a(M \beta-\alpha) v(-\alpha)=\sum_{\beta \in \mathbb{Z}^{s}} p(-\beta) w(\beta),
\end{aligned}
$$

where $w:=T_{a} v^{*}$ with $v^{*}$ given by $v^{*}(\alpha)=v(-\alpha), \alpha \in \mathbb{Z}^{s}$. Since $V_{k}$ is invariant under $T_{a}$ and $v^{*} \in V_{k}$, we have $w \in V_{k}$. It follows that

$$
\langle u, v\rangle=\sum_{\beta \in \mathbb{Z}^{s}} p(-\beta) w(\beta)=0 .
$$

For a multi-index $\mu$ with $|\mu|=k+1$, we have $\nabla^{\mu} \delta_{\alpha} \in V_{k}$ for all $\alpha \in \mathbb{Z}^{s}$. Hence $\left\langle u, \nabla^{\mu} \delta_{\alpha}\right\rangle=0$. In other words, $\nabla^{\mu} u(\alpha)=0$ for all $\alpha \in \mathbb{Z}^{s}$ and $|\mu|=k+1$. This shows that $u$ is a polynomial sequence of degree at most $k$. 
$(\mathrm{c}) \Rightarrow(\mathrm{a})$ : For $p \in \Pi_{k}$, let $q(\gamma):=\sum_{\beta \in \mathbb{Z}^{s}} a(M \beta+\gamma) p(M \beta+\gamma)$ for $\gamma \in \mathbb{Z}^{s}$. We claim that $q$ is a polynomial sequence. Indeed, by using Taylor's formula, we have

$$
p(M \beta+\gamma)=\sum_{|\mu| \leq k} t_{\mu}(M \beta) \gamma^{\mu}
$$

where $t_{\mu}:=D^{\mu} p / \mu$ !. Set $q_{\mu}(\beta):=t_{\mu}(-M \beta)$ for $\beta \in \mathbb{Z}^{s}$. Then for $\gamma \in \mathbb{Z}^{s}$,

$$
\begin{aligned}
q(\gamma) & =\sum_{\beta \in \mathbb{Z}^{s}} a(M \beta+\gamma) p(M \beta+\gamma) \\
& =\sum_{\beta \in \mathbb{Z}^{s}} \sum_{|\mu| \leq k} a(\gamma+M \beta) q_{\mu}(-\beta) \gamma^{\mu}=\sum_{|\mu| \leq k}\left(S_{a} q_{\mu}\right)(\gamma) \gamma^{\mu} .
\end{aligned}
$$

Note that $q_{\mu}$ is a polynomial sequence of degree at most $k$. By condition (c), $S_{a} q_{\mu}$ is a polynomial sequence; hence so is $q$. We observe that $q(\gamma+M \eta)=q(\gamma)$ for all $\eta \in \mathbb{Z}^{s}$ and $\gamma \in \mathbb{Z}^{s}$, that is, $q$ is a constant sequence on the lattice $\gamma+M \mathbb{Z}^{s}$ for each $\gamma \in \mathbb{Z}^{s}$. Hence $q$ itself must be a constant sequence. This verifies condition (a).

Finally, the equivalence between (a) and (d) was proved in Lemma 3.3.

We remark that the equivalence between (c) and (d) was proved in [7, p. 98] for the case when the dilation matrix $M$ is 2 times the identity matrix.

\section{SMOOTHNESS AND APPROXIMATION ORDER}

In this section we discuss the relationship between approximation and smoothness properties of a refinable function.

Suppose $\phi$ satisfies the refinement equation (1.1) with the dilation matrix $M$ being 2 times the identity matrix. It was proved by Jia in [18] that $\phi \in W_{1}^{k}\left(\mathbb{R}^{s}\right)$ and $\hat{\phi}(0) \neq 0$ imply that $\Pi_{k} \subset \mathbb{S}(\phi)$ and $\mathbb{S}(\phi)$ provides approximation order $k+1$. This result improves an earlier result of Cavaretta, Dahmen, and Micchelli about polynomial reproducibility of smooth refinable functions (see [7, p. 158]).

The above results can be extended to the case in which the dilation matrix is isotropic. Let $M$ be an $s \times s$ matrix with its entries in $\mathbb{C}$. We say that $M$ is isotropic if $M$ is similar to a diagonal matrix $\operatorname{diag}\left\{\lambda_{1}, \ldots, \lambda_{s}\right\}$ with $\left|\lambda_{1}\right|=\cdots=\left|\lambda_{s}\right|$. For example, for $a, b \in \mathbb{R}$, the matrix

$$
\left(\begin{array}{cc}
a & -b \\
b & a
\end{array}\right)
$$

is isotropic. Obviously, a matrix $M$ is isotropic if and only if its transpose $M^{T}$ is isotropic.

Lemma 6.1. Let $M$ be an isotropic matrix with spectral radius $\sigma$. For any vector norm $\|\cdot\|$ on $\mathbb{R}^{s}$, there exist two positive constants $C_{1}$ and $C_{2}$ such that the inequalities

$$
C_{1} \sigma^{n}\|v\| \leq\left\|M^{n} v\right\| \leq C_{2} \sigma^{n}\|v\|
$$

hold true for every positive integer $n$ and every vector $v \in \mathbb{R}^{s}$.

Proof. Since $M$ is isotropic, we can find a basis $\left\{v_{1}, \ldots, v_{s}\right\}$ for $\mathbb{C}^{s}$ such that $M v_{j}=$ $\lambda_{j} v_{j}$ with $\left|\lambda_{1}\right|=\cdots=\left|\lambda_{s}\right|=\sigma$. Recall that two norms on a finite-dimensional linear space are equivalent. Hence there exist two positive constants $C_{1}$ and $C_{2}$ such that

$$
C_{1} \sum_{j=1}^{s}\left|a_{j}\right| \leq\|v\| \leq C_{2} \sum_{j=1}^{s}\left|a_{j}\right| \quad \text { for } v=\sum_{j=1}^{s} a_{j} v_{j} .
$$


But for $v=\sum_{j=1}^{s} a_{j} v_{j}$ we have $M^{n} v=\sum_{j=1}^{s} a_{j} \lambda_{j}^{n} v_{j}$. It follows that

$$
\left\|M^{n} v\right\| \leq C_{2} \sum_{j=1}^{s}\left|a_{j} \lambda_{j}^{n}\right|=C_{2} \sigma^{n} \sum_{j=1}^{s}\left|a_{j}\right| \leq C_{2} C_{1}^{-1} \sigma^{n}\|v\|
$$

and

$$
\left\|M^{n} v\right\| \geq C_{1} \sum_{j=1}^{s}\left|a_{j} \lambda_{j}^{n}\right|=C_{1} \sigma^{n} \sum_{j=1}^{s}\left|a_{j}\right| \geq C_{1} C_{2}^{-1} \sigma^{n}\|v\| .
$$

This completes the proof of the lemma.

Lemma 6.2. Let $M$ be an isotropic matrix with spectral radius $\sigma$. For an infinitely differentiable function $f$ on $\mathbb{R}^{s}$, let

$$
f_{n}(\xi):=f\left(\left(M^{T}\right)^{n} \xi\right), \quad \xi \in \mathbb{R}^{s}, \quad n=0,1,2, \ldots .
$$

Then, for each positive integer $r$, there exists a positive constant $C$ depending only on $r$ and the matrix $M$ such that

$$
\max _{|\mu|=r}\left|D^{\mu} f_{n}(\xi)\right| \leq C \sigma^{r n} \max _{|\nu|=r}\left|D^{\nu} f\left(\left(M^{T}\right)^{n} \xi\right)\right| \quad \forall \xi \in \mathbb{R}^{s} .
$$

Proof. Let $B=\left(b_{p q}\right)_{1 \leq p, q \leq s}$ be the matrix $\left(M^{T}\right)^{n}$. By the chain rule, for $j=$ $1, \ldots, s$, we have

$$
D_{j} f_{n}(\xi)=\left(b_{1 j} D_{1}+\cdots+b_{s j} D_{s}\right) f\left(\left(M^{T}\right)^{n} \xi\right), \quad \xi \in \mathbb{R}^{s} .
$$

Hence, for a multi-index $\mu=\left(\mu_{1}, \ldots, \mu_{s}\right)$ with $|\mu|=r$,

$$
D^{\mu} f_{n}(\xi)=\prod_{j=1}^{s} D_{j}^{\mu_{j}} f_{n}(\xi)=\prod_{j=1}^{s}\left(b_{1 j} D_{1}+\cdots+b_{s j} D_{s}\right)^{\mu_{j}} f\left(\left(M^{T}\right)^{n} \xi\right), \quad \xi \in \mathbb{R}^{s} .
$$

By Lemma 6.1, there exists a constant $C_{1}>0$ depending only on the matrix $M$ such that $\left|b_{p q}\right| \leq C_{1} \sigma^{n}$ for all $p, q$. We may express $\prod_{j=1}^{s}\left(b_{1 j} D_{1}+\cdots+b_{s j} D_{s}\right)^{\mu_{j}}$ as $\sum_{|\nu|=r} c_{\nu} D^{\nu}$, where each $c_{\nu}$ is a linear combination of products of $r$ factors of the $b_{p q}$ 's. Hence there exists a positive constant $C$ depending only on $r$ and the matrix $M$ such that $\left|c_{\nu}\right| \leq C \sigma^{r n}$ for all $|\nu|=r$. This proves (6.1).

Now we are in a position to establish the main result of this section.

Theorem 6.3. Suppose $M$ is an $s \times s$ isotropic dilation matrix, and $a$ is an element in $\ell_{0}\left(\mathbb{Z}^{s}\right)$ satisfying (1.2). Let $\phi$ be the normalized solution of the refinement equation (1.1). If $\phi \in W_{1}^{k}\left(\mathbb{R}^{s}\right)$, then $\Pi_{k} \subset \mathbb{S}(\phi)$ and $\mathbb{S}(\phi)$ provides approximation order $k+1$.

Proof. Since $\hat{\phi}(0)=1$, in order to prove $\mathbb{S}(\phi) \supset \Pi_{k}$, it suffices to show that for $|\mu| \leq k$,

$$
D^{\mu} \hat{\phi}(2 \pi \beta)=0 \quad \forall \beta \in \mathbb{Z}^{s} \backslash\{0\} .
$$

The proof proceeds with induction on $|\mu|$, the length of $\mu$.

Let $H$ be the function given in (3.2). A repeated application of (3.1) yields that, for $n=1,2, \ldots$,

$$
\hat{\phi}(\xi)=\left[\prod_{j=1}^{n} H\left(\left(M^{T}\right)^{-j} \xi\right)\right] \hat{\phi}\left(\left(M^{T}\right)^{-n} \xi\right), \quad \xi \in \mathbb{R}^{s} .
$$


It follows that

$$
\hat{\phi}\left(\left(M^{T}\right)^{n} \xi\right)=h_{n}(\xi) \hat{\phi}(\xi), \quad \xi \in \mathbb{R}^{s},
$$

where $h_{n}(\xi):=\prod_{j=1}^{n} H\left(\left(M^{T}\right)^{j-1} \xi\right)$. Note that $H$ is $2 \pi$-periodic and $H(0)=1$. Thus, we have

$$
\hat{\phi}\left(2 \pi\left(M^{T}\right)^{n} \beta\right)=\left[\prod_{j=1}^{n} H\left(2 \pi\left(M^{T}\right)^{j-1} \beta\right)\right] \hat{\phi}(2 \beta \pi)=\hat{\phi}(2 \beta \pi), \quad \beta \in \mathbb{Z}^{s} .
$$

If $\phi \in L_{1}\left(\mathbb{R}^{s}\right)$, then by the Riemann-Lebesgue lemma we obtain

$$
\hat{\phi}(2 \beta \pi)=\lim _{n \rightarrow \infty} \hat{\phi}\left(2 \pi\left(M^{T}\right)^{n} \beta\right)=0 \quad \forall \beta \in \mathbb{Z}^{s} \backslash\{0\} .
$$

This establishes (6.2) for $\mu=0$.

Let $0<r \leq k$. Assume that (6.2) has been proved for $|\mu|<r$. We wish to establish (6.2) for $|\mu|=r$. For this purpose, we deduce from (6.3) that

$$
\hat{\phi}(\xi)=f_{n}(\xi)\left[1 / h_{n}(\xi)\right], \quad \xi \in \mathbb{R}^{s},
$$

where $f_{n}(\xi):=\hat{\phi}\left(\left(M^{T}\right)^{n} \xi\right), \xi \in \mathbb{R}^{s}$. By using the Leibniz formula for differentiation, we get

$$
D^{\mu} \hat{\phi}(\xi)=\sum_{\nu \leq \mu}\left(\begin{array}{l}
\mu \\
\nu
\end{array}\right) D^{\nu} f_{n}(\xi) D^{\mu-\nu}\left[1 / h_{n}\right](\xi), \quad \xi \in \mathbb{R}^{s} .
$$

But, for $\beta \in \mathbb{Z}^{s} \backslash\{0\}$ and $|\nu|<r$, we have $D^{\nu} f_{n}(2 \pi \beta)=0$, by the induction hypothesis. When $\nu=\mu$, we have $\left[1 / h_{n}\right](2 \pi \beta)=1$. Hence it follows from (6.4) that

$$
D^{\mu} \hat{\phi}(2 \pi \beta)=D^{\mu} f_{n}(2 \pi \beta), \quad \beta \in \mathbb{Z}^{s} \backslash\{0\} .
$$

By Lemma 6.2, we have

$$
\left|D^{\mu} f_{n}(2 \pi \beta)\right| \leq C \sigma^{r n} \max _{|\nu|=r}\left|D^{\nu} \hat{\phi}\left(\left(M^{T}\right)^{n} 2 \pi \beta\right)\right|, \quad \beta \in \mathbb{Z}^{s} \backslash\{0\},
$$

where $C>0$ is a constant independent of $n$.

In what follows, we use $v_{j}$ to denote the $j$ th coordinate of a vector $v$ in $\mathbb{R}^{s}$. For a multi-index $\nu=\left(\nu_{1}, \ldots, \nu_{s}\right)$, let $\phi_{\nu}$ be the function given by $\phi_{\nu}(x)=(-i x)^{\nu} \phi(x)$, $x \in \mathbb{R}^{s}$. Then $D^{\nu} \hat{\phi}=\hat{\phi}_{\nu}$ and

$$
\left(\left(-i D_{j}\right)^{r} \phi_{\nu}\right)^{\wedge}(\xi)=\xi_{j}^{r} D^{\nu} \hat{\phi}(\xi), \quad \xi=\left(\xi_{1}, \ldots, \xi_{s}\right) \in \mathbb{R}^{s} .
$$

Since $\phi \in W_{1}^{k}\left(\mathbb{R}^{s}\right)$, we have $\left(-i D_{j}\right)^{r} \phi_{\nu} \in L_{1}\left(\mathbb{R}^{s}\right)$. Thus, by the Riemann-Lebesgue lemma, we obtain

$$
\lim _{n \rightarrow \infty}\left(\left(M^{T}\right)^{n} \beta\right)_{j}^{r} D^{\nu} \hat{\phi}\left(2 \pi\left(M^{T}\right)^{n} \beta\right)=0 \quad \text { for } \beta \in \mathbb{Z}^{s} \backslash\{0\} .
$$

This is true for $j=1, \ldots, s$; hence it follows that

$$
\lim _{n \rightarrow \infty}\left\|\left(M^{T}\right)^{n} \beta\right\|^{r} D^{\nu} \hat{\phi}\left(2 \pi\left(M^{T}\right)^{n} \beta\right)=0 \quad \text { for } \beta \in \mathbb{Z}^{s} \backslash\{0\},
$$

where $\|\cdot\|$ is a vector norm on $\mathbb{R}^{s}$. By Lemma 6.1 , there exists a positive constant $C_{1}>0$ independent of $n$ such that

$$
C_{1} \sigma^{n}\|\beta\| \leq\left\|\left(M^{T}\right)^{n} \beta\right\| .
$$


Therefore

$$
\lim _{n \rightarrow \infty} \sigma^{n r} D^{\nu} \hat{\phi}\left(2 \pi\left(M^{T}\right)^{n} \beta\right)=0 \quad \text { for } \beta \in \mathbb{Z}^{s} \backslash\{0\} .
$$

This in connection with (6.5) and (6.6) tells us that $D^{\mu} \hat{\phi}(2 \pi \beta)=0$ for $|\mu|=r$ and $\beta \in \mathbb{Z}^{s} \backslash\{0\}$. The proof of the theorem is complete.

Recall that $\Omega$ is a complete set of representatives of the distinct cosets of $\mathbb{Z}^{s} / M^{T} \mathbb{Z}^{s}$. Thus, as a consequence of Theorem 6.3 , we conclude that if the normalized solution $\phi$ of the refinement equation (1.1) lies in $W_{1}^{k}\left(\mathbb{R}^{s}\right)$, and if $N(\phi) \cap$ $\left(2 \pi\left(M^{T}\right)^{-1} \Omega\right)=\emptyset$, then the refinement mask $a$ satisfies all the conditions in Theorem 5.2 .

\section{REFERENCES}

[1] J. Barros-Neto, An Introduction to the Theory of Distributions, Marcel Dekker, New York, 1973. MR 57:1113

[2] C. de Boor, The polynomials in the linear span of integer translates of a compactly supported function, Constr. Approx. 3 (1987), 199-208. MR 88e:41054

[3] C. de Boor, R. DeVore, and A. Ron, Approximation from shift-invariant subspaces of $L_{2}\left(\mathbb{R}^{d}\right)$, Trans. Amer. Math. Soc. 341 (1994), 787-806. MR 94d:41028

[4] C. de Boor and K. Höllig, Approximation order from bivariate $C^{1}$-cubics: a counterexample, Proc. Amer. Math. Soc. 87 (1983), 649-655. MR 84j:41014

[5] C. de Boor, K. Höllig, and S. Riemenschneider, Box Splines, Springer-Verlag, New York, 1993. MR 94k:65004

[6] C. de Boor and R. Q. Jia, A sharp upper bound on the approximation order of smooth bivariate pp functions, J. Approx. Theory 72 (1993), 24-33. MR 94e:41012

[7] A. S. Cavaretta, W. Dahmen, and C. A. Micchelli, Stationary Subdivision, Memoirs of Amer. Math. Soc., vol. 93, no. 453, 1991. MR 92h:65017

[8] I. Daubechies and J. C. Lagarias, Two-scale difference equations: II. Local regularity, infinite products of matrices and fractals, SIAM J. Math. Anal. 23 (1992), 1031-1079. MR 93g:39001

[9] R. DeVore, B. Jawerth, and V. Popov, Compression of wavelet decompositions, Amer. J. Math. 114 (1992), 737-785. MR 94a:42045

[10] T. N. T. Goodman, C. A. Micchelli, and J. D. Ward, Spectral radius formulas for subdivision operators, Recent Advances in Wavelet Analysis (L. L. Schumaker and G. Webb, eds.), Academic Press, 1994, pp. 335-360. MR 94m:47076

[11] K. Gröchenig and W. R. Madych, Multiresolution analysis, Haar bases, and self-similar tilings of $\mathbb{R}^{n}$, IEEE Transactions on Information Theory 38 (1992), 556-568. MR 93i:42001

[12] B. Han and R. Q. Jia, Multivariate refinement equations and subdivision schemes, manuscript.

[13] C. Heil, G. Strang, and V. Strela, Approximation by translates of refinable functions, Numer. Math. 73 (1996), 75-94. MR 97c:65033

[14] R. Q. Jia, A dual basis for the integer translates of an exponential box spline, Rocky Mountain J. Math. 23 (1993), 223-242. MR 94a:41022

[15] R. Q. Jia, A Bernstein type inequality associated with wavelet decomposition, Constr. Approx. 9 (1993), 299-318. MR 94h:41026

[16] R. Q. Jia, The Toeplitz theorem and its applications to Approximation Theory and linear PDE's, Trans. Amer. Math. Soc. 347 (1995), 2585-2594. MR 95i:41014

[17] R. Q. Jia, Refinable shift-invariant spaces: from splines to wavelets, Approximation Theory VIII (C. K. Chui and L. L. Schumaker, eds.), vol. 2, World Scientific Publishing Co., Inc., 1995, pp. 179-208.

[18] R. Q. Jia, The subdivision and transition operators associated with a refinement equation, Advanced Topics in Multivariate Approximation (F. Fontanella, K. Jetter and P.-J. Laurent, eds.), World Scientific Publishing Co., Inc., 1996, pp. 139-154.

[19] R. Q. Jia and C. A. Micchelli, On linear independence of integer translates for a finite number of functions, Proc. Edinburgh Math. Soc. 36 (1993), 69-85. MR 94e:41044 
[20] R. Q. Jia and C. A. Micchelli, Using the refinement equation for the construction of prewavelets V: extensibility of trigonometric polynomials, Computing 48 (1992), 61-72. MR 94a: 42049

[21] A. Ron, A characterization of the approximation order of multivariate spline spaces, Studia Math. 98 (1991), 73-90. MR 92g:41017

Department of Mathematical Sciences, University of Alberta, Edmonton, Alberta, CANADA T6G 2G1

E-mail address: jia@xihu.math.ualberta.ca 